\title{
$\sqrt{-1} \frac{\text { EasyChair Preprint }}{\text { № } 4261}$
}

\section{A Beamforming Method Based on Polarization Matching}

Xiaohui Yang, Jie Gu, Jingfei Jiang and Yunhao Li 


\title{
A Beamforming Method Based on Polarization Matching
}

\author{
Xiaohui Yang; Jie Gu; Jingfei Jiang; Yunhao Li \\ Science and Technology on Electronic Information \\ Control Laboratory \\ Chengdu, China \\ yangxiaohui0809@126.com
}

\begin{abstract}
By analyzing the polarization-sensitive array model and combining the polarization information of different elements with space-time processing, a beamforming method based on polarization matching is proposed, which realizes the high-performance beamforming of the polarization-sensitive antenna array. The simulation performance comparison with traditional spatial beamforming confirms the high direction finding accuracy and high signal-to-noise ratio robustness of this algorithm.
\end{abstract}

Keywords-Beamforming; Polarization matching; Accurate direction finding; Array signal processing

\section{INTRODUCTION}

The beam characteristics of traditional digital beamforming systems are controlled by weighted vectors and have a fixed polarization form. Such signal processing is spatial signal processing. When the arrival angles of the desired signal and the interference signal are very close, spatial filtering cannot effectively distinguish them. In addition, in the case of polarization mismatch, the signal response of some polarization forms that the array can receive is very small and cannot be effectively detected [1][3]. Therefore, the traditional array beamforming has been unable to deal with the complex electromagnetic environment of the modern battlefield.

The polarization-spatial beamforming technology that combines polarization domain information and spatial information can effectively overcome the deficiencies of spatial filtering and improve the performance of beamforming. The polarization direction of each element in the polarization-sensitive array [4] is different, and different elements will sample different polarization components of the incident signal, so the polarization-sensitive array exhibits polarization diversity. After the polarization information is introduced into the array, two-dimensional beam forming and filtering in the spatial and polarization domains can be realized. For the polarization-sensitive array, due to its two-dimensional sampling capability for the spatial and polarization domain information of the incident signal, its measurement model is more complicated than that of the polarization-consistent array, and it will have better array processing performance.

This article mainly focuses on the research on the beamforming of the polarization sensitive array in the case of polarization matching. According to the spatial geometric relationship of the polarization array radar and the theory of electromagnetic wave propagation, the receiving signal model of the polarization sensitive array is established. Then, the mechanism of realizing polarization-space combined beamforming based on polarization matching is described. Finally, the polarization matching array beamforming diagram and the estimated results of incident angle of arrival are obtained. Through comparative analysis with the results of traditional spatial beamforming, the high direction finding accuracy and high-noise robustness of the beamforming algorithm based on polarization matching are verified.

\section{PROBLEM DESCRIPTION}

\section{A. Received signal model}

Construct the array element receiving model [4] in the space rectangular coordinate system, as shown in Fig.1. If the azimuth angle of the incident signal is $\varphi$, and the elevation angle is $\theta$, the three-dimensional direction vector of the incident signal is:

$$
\mathbf{u}=\left[\begin{array}{c}
\cos \theta \sin \varphi \\
\cos \theta \cos \varphi \\
\sin \theta
\end{array}\right]
$$

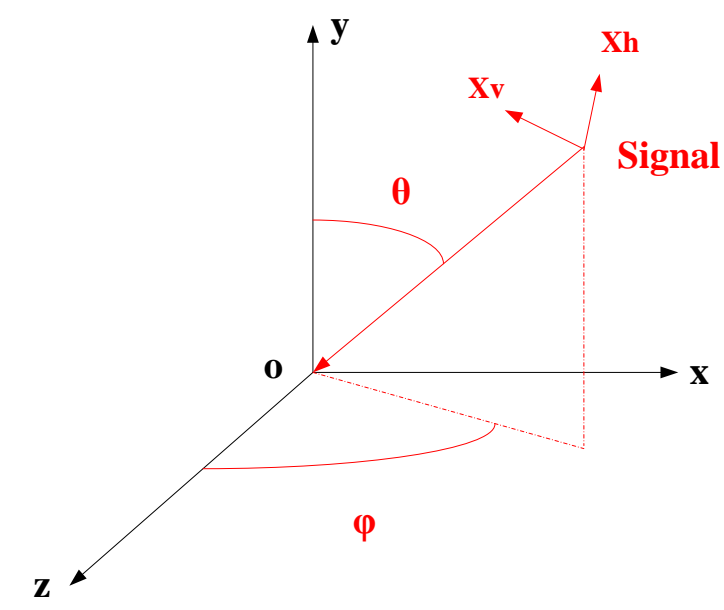

Figure 1. Schematic diagram of electromagnetic wave propagation

The expression of the space-oriented vector of any array element located at $[\mathrm{x}, \mathrm{y}, \mathrm{z}]$ is 


$$
\mathbf{a}=e^{j 2 \pi \frac{[\mathrm{x}, \mathrm{y}, \mathrm{z}] \mathbf{u}}{\lambda}}
$$

If the polarization sensitive array is composed of $\mathrm{N}$ mutually orthogonal electric dipole pairs, according to the coordinate transformation theory ${ }^{[4][5]}$, the orthogonal projection matrix in the isophase plane which perpendicular to the signal propagation direction can be expressed as:

$$
\mathbf{v}(\theta, \varphi)=\left[\begin{array}{cc}
-\cos \varphi & \sin \theta \sin \varphi \\
\sin \varphi & \sin \theta \cos \varphi \\
0 & -\cos \theta
\end{array}\right]
$$

If the polarization amplitude angle of the incident signal $\mathbf{x}(t)$ is $\gamma$, and the polarization phase angle is $\eta$, the polarization component ${ }^{[4]}$ in the two orthogonal directions of vertical and horizontal can be expressed as:

$$
\mathbf{p}(\gamma, \eta)=\left[\begin{array}{c}
\cos \gamma \\
\sin \gamma e^{j \eta}
\end{array}\right]
$$

Then, the electric field component ${ }^{[5]}$ of the incident signal $\mathbf{x}(t)$ in the rectangular coordinate system can be expressed as:

$$
\begin{aligned}
& \mathbf{E}=\mathbf{v}(\theta, \varphi) \mathbf{p}(\gamma, \eta) \mathbf{x}(t) \\
& =\mathbf{B}(\theta, \varphi, \gamma, \eta) \mathbf{x}(t)=\left[\begin{array}{cc}
-\cos \varphi & \sin \theta \sin \varphi \\
\sin \varphi & \sin \theta \cos \varphi \\
0 & -\cos \theta
\end{array}\right]\left[\begin{array}{c}
\cos \gamma \\
\sin \gamma e^{j \eta}
\end{array}\right] \mathbf{x}(t)
\end{aligned}
$$

Therefore, for an antenna with an azimuth angle of $\varphi$, an elevation angle of $\theta$, a polarization angle of $[\gamma, \eta]$, and array element pointing angles of $\alpha$ and $\beta$, when the incident signal is $\mathbf{x}(t)$, the response of the polarization sensitive array ${ }^{[4][5]}$ to the incident signal is:

$$
\mathbf{y}(\mathrm{t})=\mathbf{A x}(\mathrm{t})+\mathbf{n}(\mathrm{t})
$$

where $\mathbf{n}$ represents the noise vector, $\mathbf{A}$ is the polarizationspatial joint steering vector of the incident signal, which is the Kronecker product of the polarization steering vector $\mathbf{B}$ and the spatial steering vector $\mathbf{a}$,

$$
\mathbf{A}(\theta, \varphi, \gamma, \eta)=\mathbf{a} \otimes \mathbf{B}
$$

For an array composed of $\mathrm{N}$ array elements, if the incident waveform is $\mathbf{x}(t)$, the incident azimuth is $\varphi$, the elevation angle is $\theta$, the polarization parameter is $[\gamma, \eta]$, the received signal can be expressed as

$$
\mathbf{Y}(\mathrm{t})=\sum_{n=1}^{N} \mathbf{A}_{n}\left(\theta_{n}, \varphi_{n}, \gamma_{n}, \eta_{n}\right) \mathbf{x}(\mathrm{t})+\mathbf{N}(\mathrm{t})
$$

$\mathbf{N}(\mathrm{t})$ represents the noise vector.

\section{B. Beamforming of polarized array}

On the basis of (8) model, if there is a weighting matrix $\boldsymbol{\omega}$ that satisfies the constraint of $\boldsymbol{\omega}^{H} \mathbf{a}=\mathbf{1}$, multiply both sides of equation (8) by the weights $\omega^{H}$, then

$$
\begin{aligned}
& \boldsymbol{\omega}^{H} \mathbf{Y}=\boldsymbol{\omega}^{H} \mathbf{A} \mathbf{x}+\mathbf{N}=\mathbf{v}(\theta, \varphi) \mathbf{p}(\gamma, \eta) \mathbf{x}+\mathbf{N}, \\
& \text { s.t. } \quad \boldsymbol{\omega}^{H} \mathbf{a}=\mathbf{1}
\end{aligned}
$$

In the formula, $\square^{H}$ represents conjugate transpose. For the transmitting dual-polarized antenna, if it is known that the polarization of the antenna has $Q$ different states, namely $\left[\begin{array}{llll}\mathbf{p}_{1} & \mathbf{p}_{2} & \cdots & \mathbf{p}_{Q}\end{array}\right]$. In each known polarization state $\mathbf{p}_{q}$, if the corresponding two orthogonally polarized received signal components are $\mathbf{y}_{q i}$ and $\mathbf{y}_{q j}$, without considering the influence of noise factors, the two orthogonal components corresponding to equation (9) are:

$$
\begin{aligned}
\boldsymbol{\omega}^{H} \mathrm{y}_{q i} & =\mathbf{v}_{i}(\theta, \varphi) \mathbf{p}_{q i}(\gamma, \eta) \mathbf{x} \\
\boldsymbol{\omega}^{H} \mathrm{y}_{q j} & =\mathbf{v}_{j}(\theta, \varphi) \mathbf{p}_{q j}(\gamma, \eta) \mathbf{x}
\end{aligned}
$$

Among them, $\mathbf{v}_{i}(\theta, \varphi)$ and $\mathbf{v}_{j}(\theta, \varphi)$ represent projections in two orthogonal directions, $\mathbf{p}_{q i}$ and $\mathbf{p}_{q j}$ are polarization states in two orthogonal directions. The input signals $\mathbf{x}(t)$ in the two orthogonal directions are the same. Therefore, without considering the noise, the incident signal $\mathbf{x}(t)$ can be eliminated by (10) and (11):

$$
\frac{\omega^{H} \mathrm{y}_{q i}}{\omega^{H} \mathrm{y}_{q j}}=\frac{\mathbf{v}_{i}(\theta, \varphi) \mathbf{p}_{q i}(\gamma, \eta)}{\mathbf{v}_{j}(\theta, \varphi) \mathbf{p}_{q j}(\gamma, \eta)}
$$

(12) can be further transformed into

$$
\boldsymbol{\omega}^{H} \mathrm{y}_{q i}=\frac{\mathbf{v}_{i}(\theta, \varphi) \mathbf{p}_{q i}(\gamma, \eta)}{\mathbf{v}_{j}(\theta, \varphi) \mathbf{p}_{q j}(\gamma, \eta)} * \boldsymbol{\omega}^{H} \mathrm{y}_{q j}=\mathbf{A} \mathbf{x}_{q} * \boldsymbol{\omega}^{H} \mathrm{y}_{q j}
$$

where

$$
\mathbf{A} \mathbf{x}_{q}=\frac{\mathbf{v}_{i}(\theta, \varphi) \mathbf{p}_{q i}(\gamma, \eta)}{\mathbf{v}_{j}(\theta, \varphi) \mathbf{p}_{q j}(\gamma, \eta)}
$$

At this time, when it is known that the received signals matched by each polarization state are $\mathbf{y}_{q i}$ and $\mathbf{y}_{q j}$, the problem of solving the optimal weight $\boldsymbol{\omega}$ can be transformed into the following optimization problem of the objective function:

$$
\begin{aligned}
& \min _{\boldsymbol{\omega}}\left\|\sum_{q=1}^{Q} \boldsymbol{\omega}^{H}\left(\mathrm{y}_{q i}-\frac{\mathbf{v}_{i}(\theta, \varphi) \mathbf{p}_{q i}(\gamma, \eta)}{\mathbf{v}_{j}(\theta, \varphi) \mathbf{p}_{q j}(\gamma, \eta)} \mathrm{y}_{q j}\right)\right\|_{2} \\
& \text { s.t. } \quad \boldsymbol{\omega}^{H} \mathbf{a}=\mathbf{1}
\end{aligned}
$$

Where $\|\square\|_{2}$ represents the $l_{2}$-norm. (15) means that if there are signals incident from the direction $(\theta, \varphi)$ under $Q$ polarization states of the array change, if the two orthogonal received signals corresponding to each polarization state are $\mathbf{y}_{q i}$ and $\mathbf{y}_{q j}$, after filtering with the optimal weight $\boldsymbol{\omega}$, the output of the array beam synthesis $\mathbf{y}_{q i}$ should be the closest to the product of $\mathbf{y}_{q j}$ and $\mathbf{A} \mathbf{x}_{q}$. (15) is a quadratic programming problem with equality constraints and has an analytical solution. Using the Lagrange multiplier method, the result of (16) can be derived as

$$
\boldsymbol{\omega}=\frac{\mathbf{R} \mathbf{x}^{-1} * \mathbf{a}}{\mathbf{a}^{H} * \mathbf{R} \mathbf{x}^{-1} * \mathbf{a}}
$$

where

$$
\mathbf{R x}=\sum_{q}\left(\mathrm{y}_{q i}-\mathbf{A} \mathbf{x}_{q} \mathrm{y}_{q j}\right)^{H}\left(\mathrm{y}_{q i}-\mathbf{A} \mathbf{x}_{q} \mathrm{y}_{q j}\right)
$$

Using the analytical solution $\boldsymbol{\omega}$ in (16), traversing possible directions $(\theta, \varphi)$ and finding the energy peak point of the array output, a rough estimation of the target direction of arrival can be achieved.

\section{SIMULATION ANALYSIS}

This section tests the performance of the algorithm by performing a polarization matching-based beamforming simulation on an orthogonal dual-polarized antenna array, 
and comparing it with the traditional polarization consistent beamforming algorithm.

Assuming that the antenna array is a uniform matrix, the center frequency is $f_{c}$, the corresponding wavelength is $\lambda$, the aperture is $2 \lambda$, the array element spacing is $\lambda / 2$, the azimuth angle of the signal is $-6^{\circ}$, the elevation angle is $10^{\circ}$, the agility range of the polarization angle $\gamma$ of the incident signal is $0 \sim 90^{\circ}$ ( $1^{\circ}$ interval), the polarization phase angle $\eta$ is fixed at $0^{\circ}$, and the signal-to-noise ratio is $15 \mathrm{~dB}$. Under this condition, the simulated polarization uniform beamforming results and the polarization matching-based beamforming results described in this article are shown in Fig. 2 and Fig.3, respectively. As can be seen from Fig.2 and Fig.3, the estimated values of the incident angle of the polarization uniform beamforming algorithm and the polarization matching-based beamforming algorithm are both equal to the actual incident angles, which azimuth angle is $-6^{\circ}$ and elevation angle is $10^{\circ}$. However, the beam width of the beamforming algorithm based on polarization matching is narrower.

Fig.4 shows the cross-sectional effect of Fig.2 and Fig.3 at an azimuth of $-6^{\circ}$ and an elevation of $10^{\circ}$. From Fig.4, for the $-3 \mathrm{~dB}$ beam width, it can be seen that the azimuth and elevation angles of the uniformly polarized beam are better than $18^{\circ}$ and $17^{\circ}$, and these of the polarization matching are better than $4^{\circ}$ and $4^{\circ}$. The energy of beamforming algorithm based on polarization matching is more concentrated.

Next, in the broadband range, compare the direction finding performance of this algorithm and the polarization uniform beamforming algorithm under different signal-tonoise ratios. Under each signal-to-noise ratio, after 50 repeated experiments, the direction finding performance comparison based on polarization matching and polarization uniform beamforming is shown in Fig.5. It can be seen from the comparison result that as the signal-to-noise ratio decreases, the direction-finding errors tend to increase. Compared with the direction finding result of uniform polarization beamforming, the direction finding error based on polarization matching is significantly reduced under the same signal-to-noise ratio. Typically, when the SNR is $15 \mathrm{~dB}$, the direction finding r.m.s. of the polarization uniform beamforming method is $0.52^{\circ}$, while the direction finding r.m.s. described in this article is $0.27^{\circ}$. The beamforming algorithm based on polarization matching has higher direction finding accuracy and better robustness to signal-tonoise ratio.

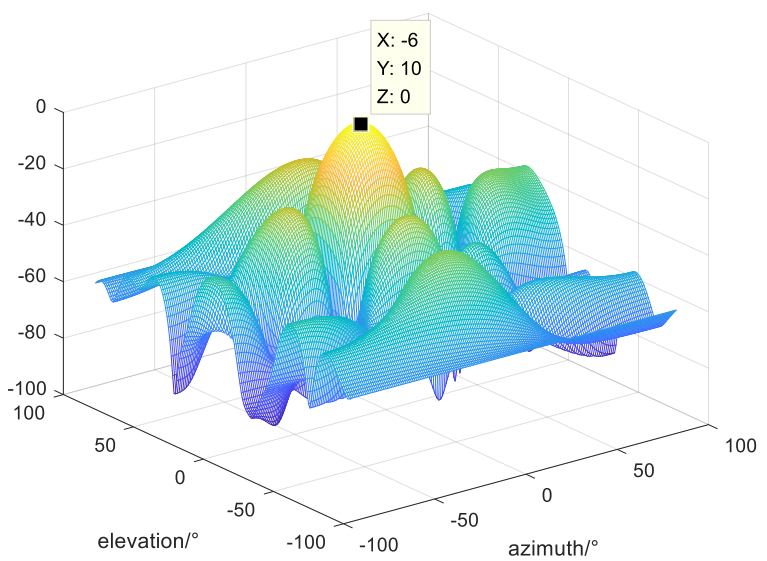

Figure 2. Beamforming with uniform polarization.

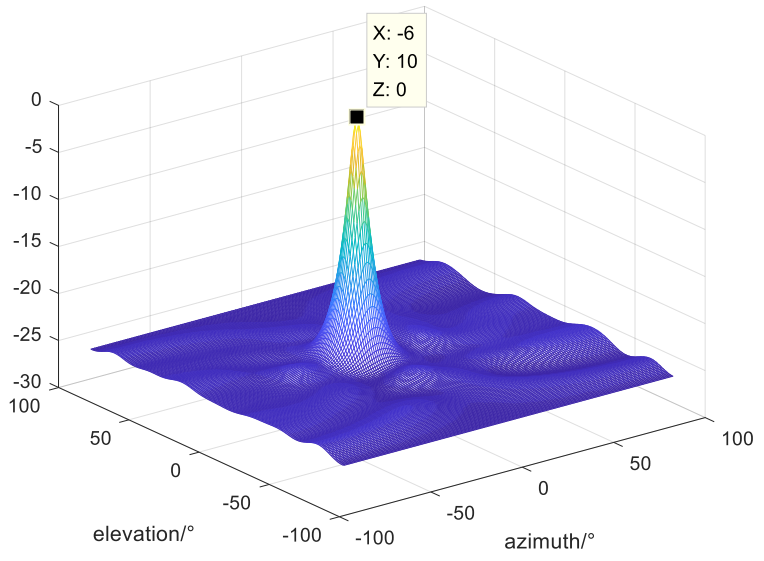

Figure 3. Beamforming with matched polarization

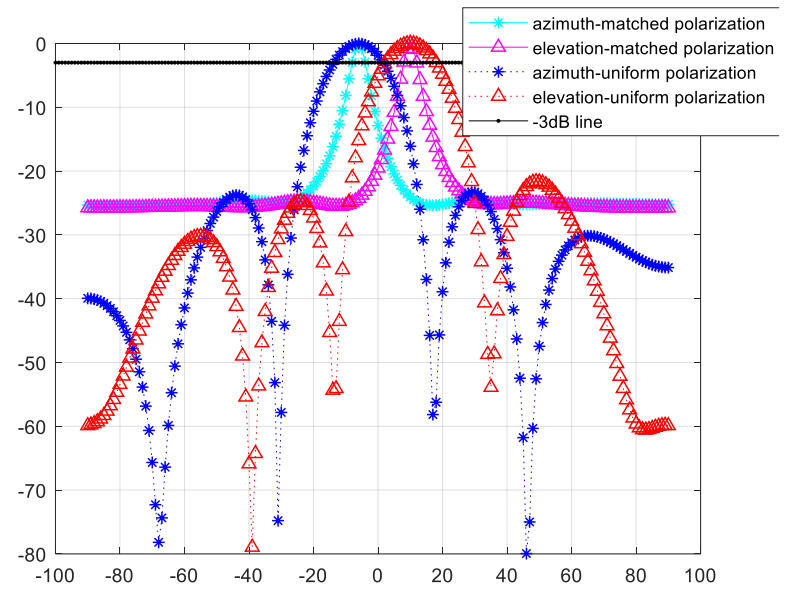

Figure 4. Comparison of beam profiles of azimuth and elevation 


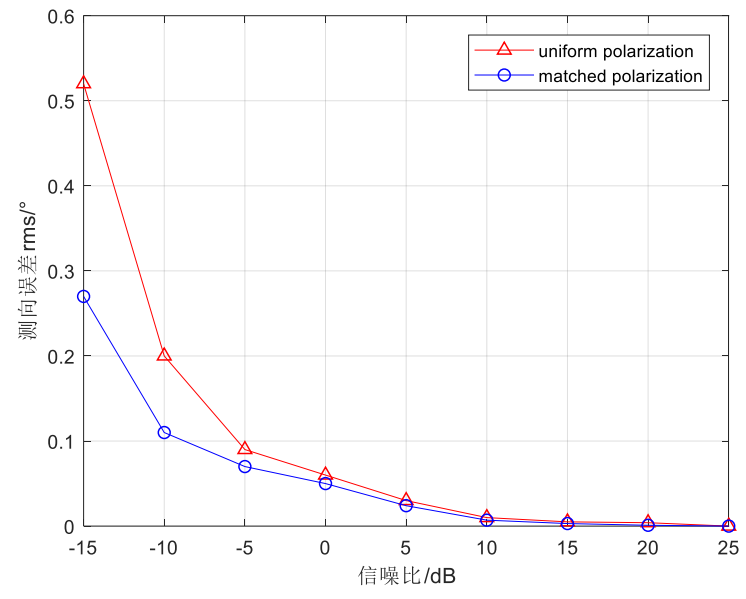

Figure 5. Statistical comparison of direction finding performance under different signal-to-noise ratios (50 repeated tests)

\section{CONCLUSION}

This paper is starting from the theoretical response of the polarization-sensitive uniform area array to the incident signal, by analyzing the polarization-sensitive array model, a beamforming algorithm in the case of polarization matching is constructed. Through simulation and comparative analysis with traditional beamforming algorithms, it is proved that the beam width formed by this algorithm is narrower, the direction finding accuracy is higher, and the signal-to-noise ratio is more robust.

\section{REFERENCES}

[1] AHMAD M. I., LIU Z. W. and XU Y. G., "Mininum variance beamforming using polarization sensitive array[C]", Communication Systems, IEEE ICCS, Guang-zhou, 2008:489-492.

[2] Yougen $\mathrm{Xu}$;Jingyan $\mathrm{Ma}$, "Polarization sensitive PARAFAC beamforming for near-field/far-field signals using co-centered orthogonal loop and dipole pairs", IEEE China Summit and International Conference, 10.1109/ChinaSIP.2013.6625415.

[3] Hui Chen;Huaizong Shao;Wenqin Wang, "Sparse reconstructionbased angle-range-polarization-dependent beamforming with polarization sensitive frequency diverse array", IEEE International Conference on Acoustics, 10.1109/ICASSP.2016.7472206.

[4] Kainam Thomas Wong, Michael D. Zoltowski, "Closed-form direction finding and polarization estimation with arbitrarily spaced electromagnetic vector-sensors at unknown locations", IEEE TRANSACTIONS ON ANTENNAS AND PROPAGATION, VOL. 48, NO. 5, MAY 2000

[5] Arye Nehorai, Eytan Paldi, "Vector-sensor array processing for electromagnetic source localization", IEEE TRANSACTIONS ON SIGNAL PROCESSING, VOL. 42, NO. 2, FEBRUARY 1994 